\title{
Introduction to the Special Issue on Biophotonics-Part 1
}

W ELCOME to the IEEE JOURNAL OF SELECTED TOPICS IN QUANTUM ELECTRONICS (JSTQE) Special Issue on Biophotonics-Part 1! In the papers included in this issue, you will be introduced to the latest developments in biophotonics, one of the most extensively growing areas in biomedical technology. Clinical prospects in biophotonics are vast and exciting that include applications ranging from minimally invasive diagnostics and therapeutics to development of novel nanobiotechnologies and nanobiomaterials. Moreover, the biophotonics field has introduced opportunities for interdisciplinary research between physicists and life scientists: state-of-the-art techniques in quantum electronics, lasers, fiber optics, and electro-optics are more applicable to the life sciences and medicine than ever. Advanced biophotonics imaging, microscopy, and sensing techniques remain one of the most widely used biomedical technologies because of their advanced features. Nonionizing radiation provides minimally invasive tissue sensing and imaging in vivo with a high spatial resolution in the micrometer, submicrometer, and nanometer range beyond the diffraction limit. Recently, minimally invasive biophotonics techniques have been developed as potential alternatives to conventional medical methods for diagnostics, monitoring and therapeutics of diseases, drug discovery, proteomics, and environmental detection of biological agents. These techniques offer a noncontact, effective, fast, and painless way for sensing and monitoring various biomedical quantities. Minimally invasive biophotonic medical devices are rapidly finding their way into the mainstream for early disease diagnosis, improved patient acceptance, and comfort. Furthermore, recent research efforts and developments in the area of biophotonics technology confirm its compatibility with modern nanotechnology trends, which will lead to development of novel ultrahigh-resolution nanobioimaging and nanobiosensor modalities for precise diagnostics and therapeutics at cellular, intracellular, and molecular levels.

The objective of this JSTQE Special Issue on BiophotonicsPart 1 is to highlight recent progress and trends in novel biophotonics technology development. The papers published in this issue cover a broad range of advanced biophotonics areas summarized in the following six sections:

1) advanced bioimaging and microscopy;

2) novel approaches in biophotonic diagnostics and therapeutics;

3) photoacoustic tomography;

4) light-cell and light-tissue interactions;

5) novel biosensing techniques;

6) advanced nanobiophotonics.
These key biophotonics topics are discussed in both Invited and Contributed papers published, which provide comprehensive overviews of the current status and future trends as well as original results and recent developments in biophotonics. We hope you will find this IEEE JSTQE Special Issue on Biophotonics-Part 1 to be an interesting and useful reference that stimulates further advances in the biophotonics field.

Because of the significant interest in the biophotonics topics, the Editorial Board has planned to publish the IEEE JSTQE Special Issue on Biophotonics in two parts. Following the present JSTQE Special Issue on Biophotonics-Part 1, we will be pleased to introduce the JSTQE Special Issue on BiophotonicsPart 2, which will be published in July/August of 2010.

\section{ACKNOWLEDGMENT}

This Issue was made possible by dedicated efforts of a number of people. First, we would like to thank the authors of all the papers in this issue as well as many reviewers around the world who, despite their many other commitments, provided highquality reviews of the manuscripts. We would like to thank the IEEE publications staff for their general support, and Chin Tan Lutz, in particular, for her prompt help, boundless energy, and excellent organization skills in helping us meet the deadline. We would like to thank Dr. Fil Bartoli, Editor-in-Chief of the JOURNAL OF SPECIAL TOPICS IN QUANTUM ElECTRONICS, for his stimulating encouragement for this Special Issue.

ILKO K. ILEV, Editor

Center for Devices and Radiological Health

U.S. Food and Drug Administration

Silver Spring, MD 20993 USA

ilko.ilev@fda.hhs.gov

LIHONG V. WANG, Editor

Department of Biomedical Engineering

Washington University in St. Louis

St. Louis, MO 63130 USA

STEPHEN A. Boppart, Editor

Beckman Institute for Advanced Science and Technology Departments of Bioengineering, Electrical and Computer Engineering, and Medicine

University of Illinois at Urbana-Champaign

Urbana, IL 61801 USA

Digital Object Identifier 10.1109/JSTQE.2010.2048255 
STEFAN ANDERSSON-ENGELS, Editor

Division of Atomic Physics

Lund University

Lund, SE-22100, Sweden

\author{
BEOP-MIN KIM, Editor \\ Department of Biomedical Engineering \\ Korea University \\ Seoul 136-703, Korea
}

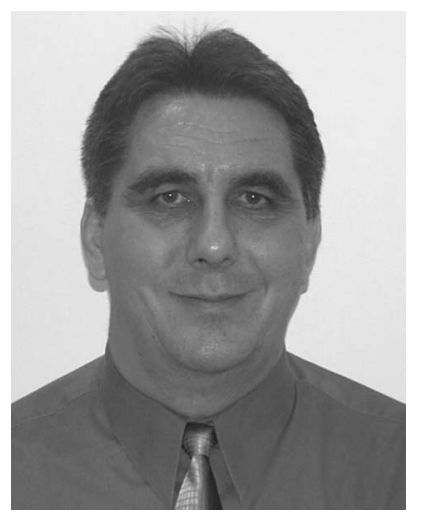

Ilko K. Ilev (M'93-SM'99) received the M.S. degree in laser physics from Sofia University, Sofia, Bulgaria, and the Ph.D. degree in laser physics from the Technical University of Sofia, Sofia, in 1983 and 1992, respectively.

He has over 20 years of experience in the United States, Europe, and Japan in the field of quantum physics and laser technologies, fiberoptics, laser medicine, noninvasive optical diagnostics and therapeutics, and biosensing and ultrahigh-resolution optical imaging. During 1995 , he was with the Optoelectronics Division, Strathclyde University, Glasgow, U.K. From 1995 to 1997, he was with the Laser Technology Laboratory, Institute of Physical and Chemical Research (RIKEN), Tokyo, Japan. From 1998 to 2001, he was an American Academy of Science/National Research Council (NRC) Research Associate in the U.S. Food and Drug Administration (FDA), Silver Spring, MD. Since May 2001, he has been with the Center for Devices and Radiological Health (CDRH), FDA, where he is the Leader of the Optical Therapeutics and Medical Nanophotonics Laboratory. He is also an Adjunct Professor in the Department of Bioengineering at the University of Maryland. He is the author or coauthor of more than 300 papers published in peer-reviewed journals and has made presentations at major national and international conferences and meetings. His current research interests include the development of novel and simple minimally invasive biophotonics and nanobiophotonics techniques including multifunctional modalities and approaches for studying light-tissue interactions at cellular, intracellular, and molecular level, and ultrahigh-resolution bioimaging and biosensing beyond the diffraction limit in the subwavelength nanoscale range.

Dr. Ilev served as an IEEE Photonics Society (formerly LEOS_Lasers and Electro-Optics Society) Biophotonics Committee Chair. He has organized and chaired conferences on biophotonics and nanobiophotonics topics within the IEEE Photonics Society, the IEEE Engineering in Medicine and Biology Society (EMBS), The International Society for Optical Engineers (SPIE), and the Optical Society of America (OSA). He serves as an Associate Editor of the IEEE TRANSACTIONS ON BIOMEDICAL ENGINEERING (TBME), a Primary Guest Editor of the IEEE JOURNAL OF SELECTED TOPICS IN QUANTUM ELECTRONICS (JSTQE) on BIOPHOTONICS, and a Guest Editor on Advanced Nanobiophotonics and Nanobiomaterials for the Nanobiotechnology Journal.

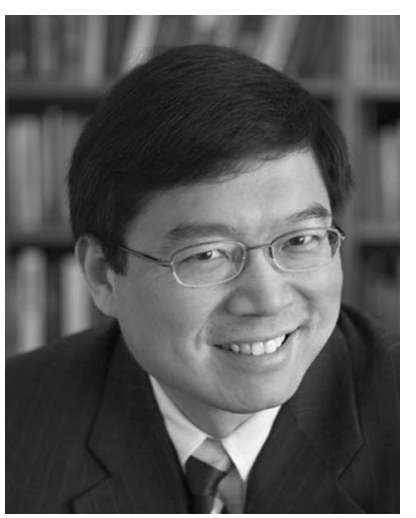

Lihong V. Wang (M'96-SM'00-F'06) worked toward the Ph.D. degree at Rice University, Houston, TX, under the tutelage of Dr. Robert Curl, Dr. Richard Smalley, and Dr. Frank Tittel.

He holds the Gene K. Beare Distinguished Professorship of biomedical engineering at Washington University in St. Louis, St. Louis, MO, and directs the Optical Imaging Laboratory. He edited the first comprehensive book on photoacoustic tomography. He is the author or coauthor of 223 peer-reviewed journal articles and has delivered 239 invited talks. His laboratory invented or discovered frequency-swept ultrasound-modulated optical tomography, dark-field confocal photoacoustic microscopy (PAM), optical-resolution PAM, photoacoustic Doppler sensing, photoacoustic reporter gene imaging, focused scanning microwave-induced thermoacoustic tomography, exact reconstruction algorithms for photoacoustic or thermoacoustic tomography, sonoluminescence tomography, Mueller-matrix optical coherence tomography, optical coherence computed tomography, and oblique-incidence reflectometry. His Monte Carlo model of photon transport in scattering media has been used worldwide.

Prof. Wang is a Fellow of the American Institute for Medical and Biological Engineering (AIMBE), the IEEE, the Optical Society of America (OSA), and The International Society for Optical Engineers (SPIE). He is the Editor-in-Chief of the Journal of Biomedical Optics. He serves as an equal Co-Chair for the Annual Conference on Photons Plus Ultrasound, the 2010 Gordon Conference on Lasers in Medicine and Biology, and the 2010 OSA Topical Meeting on Biomedical Optics. He also Co-Chairs the International Biomedical Optics Society. He is a Chartered Member on a National Institutes of Health (NIH) Study Section. He serves as the Founding Chairs of the Scientific Advisory Boards for two companies commercializing his inventions. He received an NIH FIRST Award and a National Science Foundation (NSF) CAREER Award. He authored one of the first textbooks in the field of biomedical optics and received the 2010 Joseph W. Goodman Book Writing Award. 


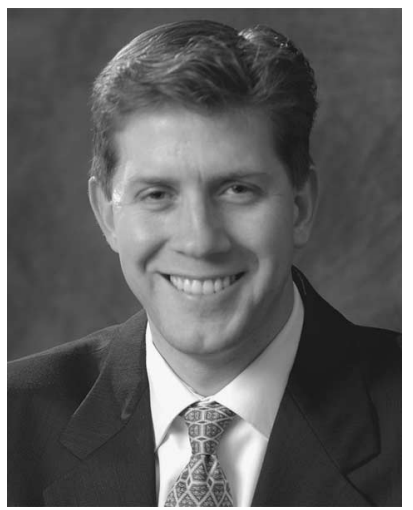

Stephen A. Boppart (S'90-M'90-SM'06) was born in Harvard, IL, in 1968. He received the B.S. degree in electrical and bioengineering and the M.S. degree in electrical engineering from the University of Illinois at Urbana-Champaign, Urbana-Champaign, in 1990 and 1991, respectively, the Ph.D. degree in electrical and medical engineering from Massachusetts Institute of Technology, Cambridge, in 1998, and the M.D. degree from Harvard Medical School, Boston, MA, in 2000.

He completed residency training in internal medicine from the University of Illinois at UrbanaChampaign in 2005. Prior to beginning his doctoral work, he was a Research Scientist at the Air Force Laser Laboratory, Brooks Air Force Base, San Antonio, TX, where he was involved in developing national [American National Standards Institute (ANSI)] and Air Force laser safety standards. Following the doctoral studies, he returned to the University of Illinois at UrbanaChampaign in 2000, where he is currently a Professor of electrical and computer engineering, bioengineering, and medicine, and Head of the Biophotonics Imaging Laboratory, Beckman Institute for Advanced Science and Technology. He also holds an appointment as Leader of a campus-wide Imaging Initiative. He is the author or coauthor of more than 165 invited and contributed publications and has made over 400 invited and contributed presentations. He is the holder of over 25 patents filed or pending. His current research interests include the development of novel optical imaging technologies for biological and medical applications, with particular emphasis on translating these to clinical applications in cancer detection and diagnosis.

Prof. Boppart is a Fellow of the Optical Society of America and The International Society for Optical Engineers (SPIE), and a member of the Society for Molecular Imaging, the Academy of Molecular Imaging, the American Association for the Advancement of Science, the American Association for Cancer Research, and the American Medical Association. In 2002, he was named one of the Top 100 Innovators in the World by the Technology Review Magazine for his research in medical technology, and, in 2005, he received the IEEE Engineering in Medicine and Biology Society (EMBS) Early Career Achievement Award.

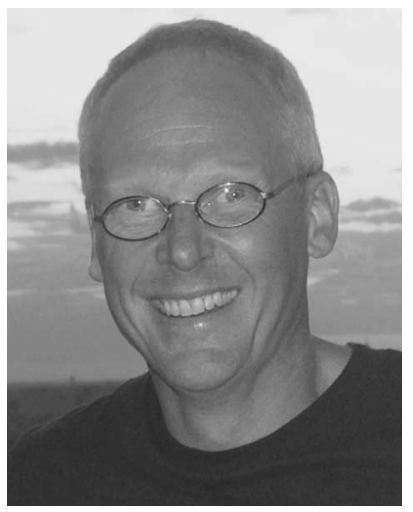

Stefan Andersson-Engels received the M.Sc. degree in engineering physics and the Ph.D. degree in physics from Lund University, Lund, Sweden, in 1985 and 1990, respectively.

He was involved in developing methods for tissue diagnostics based on optical spectroscopic techniques. He was at McMaster University, Hamilton, ON, Canada, for one year, and was involved in tissue optics as well as confocal and two-photon microscopy. In 1993, he joined Lund University as an Assistant Professor, and became an Associate Professor in 1994 and a Full Professor in 1999. He is the author or coauthor of more than 160 articles published in peerreviewed journals. His current research interests include optical spectroscopy for biomedical and pharmaceutical applications as well as interstitial photodynamic therapy of malignant tumors.

Prof. Andersson-Engels has been a Co-Organizer of several international conferences including the Gordon Conference on Lasers in Biology and Medicine in 2000, the European Conference on Biomedical Optics in 2003, and a series of biannual international summer schools in biophotonics at the Island Ven in Sweden.

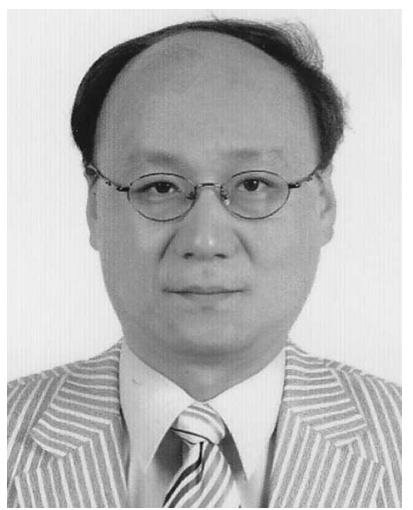

Beop-Min Kim received the B.S. degree in mechanical engineering from Korea University, Seoul, Korea, in 1989, and the M.S. and Ph.D. degrees in bioengineering from Texas A\&M University, College Station, in 1991 and 1996, respectively.

He was a Predoctoral Fellow at the University of Texas M.D. Anderson Cancer Center, Houston, and University of Texas Medical Branch at Galvaston from 1993 to 1996. From 1996 to 2001, he has been with the Medical Technology Program, Lawrence Livermore National Laboratory, CA, where he was the Staff Scientist. From 2001 to 2009, he was an Assistant/Associate Professor in the Department of Biomedical Engineering at Yonsei University, Korea, where he also served as the Department Head and the Director of the Institute of Advanced Biomedical Engineering. He is currently a Professor in the Department of Biomedical Engineering, Korea University. He is the author or coauthor of more than 40 papers in peer-reviewed journals and has made over 100 presentations at major national and international conferences and chaired many conferences on biophotonics within the Optical Society of Korea. His research interests include optical coherence tomography, near-IR spectroscopy (NIRS) in neuroscience, and second harmonic generation microscopy.

Prof. Kim is currently a Board Member of the Optical Society of Korea and the Korean Society of Medical and Biological Engineering. 